\title{
The Design of Automated Negotiation Framework for SMEs Networking
}

\author{
Li Zhuyan ${ }^{1, a}$, Zhou Guixian ${ }^{2, b}$ * \\ ${ }^{1}$ Information Institute GuiZhou College of Financial and Economic, Guiyang 550004, China \\ 2 Information Institute GuiZhou College of Financial and Economic, Guiyang 550004, China \\ a2225837531@qq.com, bhouasp1978@126.com
}

Keywords: Negotiation Framework, SMEs

Abstract. Small and Medium size Enterprises (SMEs) are considered as the backbone of the manufacturing industry in China, and a breeding ground for business ideas for the knowledge-based economy. Flexible integration of resources and engineering collaboration of self-governed SMEs is expected to facilitate the strengthening of the competitive position of SMEs. this paper designs the negotiation business model of MRMS based on ASP, brings forward users automated negotiation workflow based on agent, presents the automated negotiation mathematics expressions, and puts forward an high efficiency evaluation method of this expressions falling together utility function, multi-topics comparability calculated.

\section{Introduction}

Advancements of ISI have facilitated global sharing of valuable information, which in turn has a boosting effect on the competition of producers, resulting in structural changes in the world's economy. Transfer of manufacturing capacity from high-cost locations to low-cost countries due to competitive pressure caused by globalization, has shifted China's comparative advantage towards knowledge-based activities. Small and Medium size Enterprises (SMEs) are considered as the backbone of the China's economy, as well as a key source of jobs and a breeding ground for business ideas for the knowledge-based economy. Flexible integration of resources and engineering collaboration of self-governed SMEs is expected to facilitate the strengthening of the competitive position of SMEs in their struggle for the future through acquisition of a critical mass in terms of necessary skills, capabilities and capacities[1].

Through structuring the management database of the area manufacturing resource and modeling of manufacturing resource and effective search method, the manufacturing resource management system (MRMS) based on application service provider (ASP) have offered the basic platform to set up the dynamic alliance of enterprises swiftly and effectively for the customer. It realize sharing and conformity of manufacturing resources of the relevant enterprises in the area or the trade (including products resource, technological resource, design resource, equipment resource, human resources , market channel ,etc. ). when order enterprise have the business undertook outside and need to look for the cooperative partner of processing, MRMS based on ASP can offer a mechanism for order enterprise (customer) to search proper manufacturer that accomplish the task of processing. Concrete logic is to register a large number of clusters of manufacturer's enterprise with specific manufacturing capacity in this system. According to the demand for the task of manufacturing and distribution situation of potential manufacturers' manufacture resource and working state situation of manufacturer, order enterprise can find a set of manufacturers that can meet the require of manufacturing task actively or passively (referring to manufacturer initiative connection ) [2]. MRMS based on ASP offer a automatic negotiation mode order of enterprises and manufacturing company based on ASP necessarily, in order to improve success rate and benefit that the dynamic alliance sets up, as is showed by Fig. 1 . 


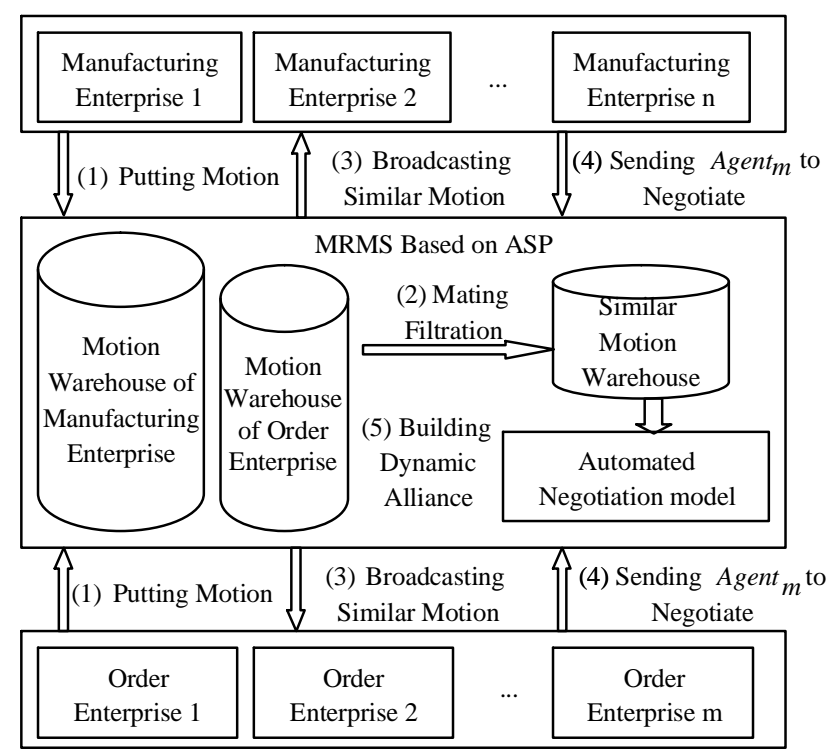

Fig. 1 the Framework of Automatic Negotiation in MRMS Based on ASP

In the framework of automatic negotiation system of manufacturing resource management based on ASP, it regard ASP platform as the center. And its business procedure is described as follows:

(1)At first manufacturing company and order enterprise propose one's own motion to system separately (for instance: name of manufacturing resource, holding resource quantity, resource state and using prices of resource etc.; name of task undertook outside, name and quantity of necessary resources and the reference price of task undertook outside etc.);

(2)In all motion warehouse of the manufacturing company(seller) and order enterprise(buyer), it screen out the motion that similar degree is higher by mating mode to make up of similar motion warehouse;

(3)In the form of notifying broadcast, the result that is screened out by mating mode is notified to the manufacturing company and order enterprise.

(4)In manufacturing company and order enterprise similar degree getting high in a one's own partnership mode separately have priority to send Agents to consult with the other side;

(5)If it succeeds to negotiate, we set up the dynamic alliance on ASP platform finally, and change over to the business operation at the next stage.

\section{NEGOTIATION BUSINESS MODEL TO DESIGN}

\section{Negotiation Procedure to Design}

Mutual reciprocity and mutual benefit tactics is a successful mode that mankind negotiate. It refer to that negotiating parties agree with barter unimportant topic that oneself feel for the more important topic that other side think for, or when both sides can refuse to budge to a certain topic, will give in each other. In mating mode of automated negotiation in MRMS based on ASP, screening the motion that the similar degree is higher from a lot of manufacturing companies and a lot of order enterprises consult the negotiation. During the process of negotiating, negotiate Agent combine the fuzzy theory and mutual reciprocity and mutual benefit tactics to realize the mechanism of automatic negotiation based on Agent[3].

In order to enable Agents to understand correctly users' whole satisfaction to different negotiating schemes (calculation which sees through mathematics function, calculate out the satisfying value), when the other side proposes the scheme, Agent calculates its satisfaction according to one's own utility function. If unsatisfied, we would propose another scheme to the other side by our Agent, until reaching an agreement or negotiation stopping.

Because negotiation belongs to negotiation structure of many topic, this research design utility function of many topic to maximize utility. In MRMS based on ASP automated negotiation use the fuzzy method to imitate the human thinking mode, and adopt the fuzzy reasoning method to find out 
the new motion in a lot of topics of negotiating cooperating with the tactics of mutual reciprocity and mutual benefit. Automated negotiation mechanism enable Agent carrying on negotiation of many attributes at the same time, and can consider the partiality of the other side in the course of negotiating, and give in to possible attribute. Designing of its negotiating procedure is showed by Fig. 2.

Every step of negotiating procedure proves as follows:

Step 0: According to screening result of mating mode at first, it enter negotiation system if it succeed to mate and consult beginning.

Step 1: Calculating out the motion of the other side apply to one's own utility value.

Step 2: Utility value calculated out from the step l compares with one's own threshold value. If it is greater than threshold value, we accept the other side's motion, and enter the step 8.

Step 3: If negotiating times $\mathrm{n} 1>\mathrm{N}$ ( $\mathrm{N}$ is negotiation times that system establishes), refuse motion and enter the step 8; If negotiating times $\mathrm{n} 1<\mathrm{N}$, then enter the step 4.

Step 4: It calculates and produces the new motion to combine utility function and fuzzy reasoning method.

Step 5: New motion produced by step 4 calculates value of $\lambda\left(\lambda=\min \left\{U^{S}\left(x^{S}\right), \operatorname{Sim}\left(x^{S}, x^{B}\right)\right\}\right)$, if value of $\lambda$ is greater than the value of the last motion, then get back to step 1 and continue negotiating.

Step 6: If the value of $\lambda$ is smaller than the value of the last motion, and $N 2<M$ (M is the times that the system products new motion and establishes), then return the step 4 and find new motion newly.

Step 7: If N2>M, refuse motion and enter the step 8.

Step 8: After it over to negotiate, the negotiating result (accepting and refusing motion) notifies manufacturing company and both sides of order enterprise finally.

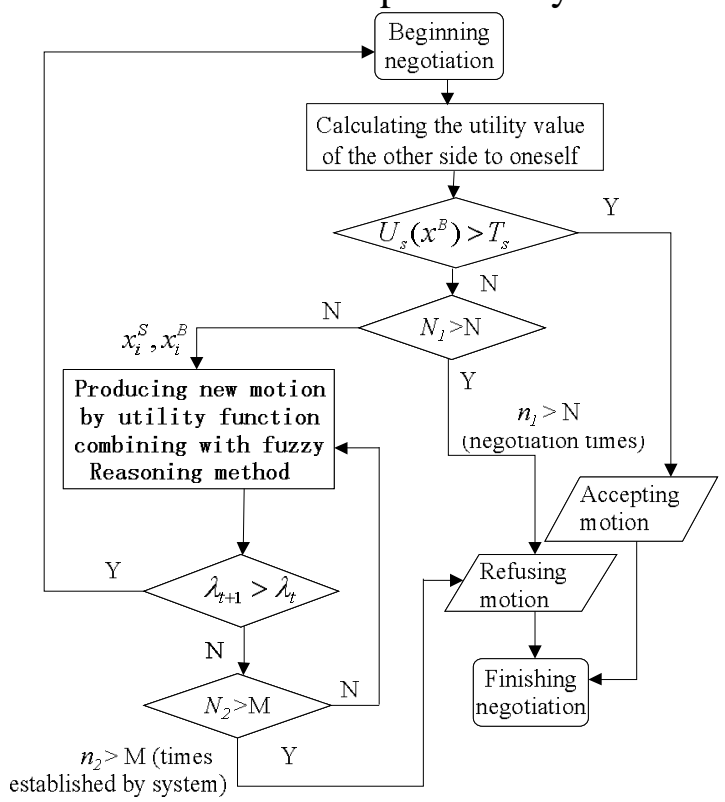

Fig.2 Flow of Negotiation

Efficient unified management, reduce working intensity of management personnel

Desktop virtualization platform through the desktop computing resource pooling management, implement flexible rapid deployment, on demand. For desktop deployment and distribution, the administrator only on making image template management stage, after a batch produced and restart dozens or even hundreds of desktop, and the whole process but over ten minutes. After pooling resources management, can open desktop resources in resource utilization is low for students with special needs, time and billing, do according to the need to use, pay by using, make full use of IT resources.

Desktop virtualization as a way of cloud computing, the requirement to the terminal equipment is greatly reduced, can use a variety of computing terminal access to use desktop virtualization platform for virtual desktop. Desktop virtualization platform based on server computing architecture greatly 
reduce the computing needs of front-end equipment, so as to prolong the service life of the original PC terminal, with the elimination of old equipment can keep monitor continue to use, save large amount of desktop terminal input costs[4].

\section{conclusion}

With the rapid development of global manufacturing industry, network manufacturing system will find a wider application. This research develops the case ASP platform which matchs the market regulations, and conforms a batch of application services. The case ASP platform offers the features of HR, SCM, CRM, DRP etc. In this paper we survey the research in the area of ASP. We also analyze business environment and discusses the MIS design and implementation of the proposed platform based on ASP. This paper Based on the demands on generality, comprehensiveness, openness, practicality and superiority, developed the structure of ASP network manufacturing system, the general plan for ASP network manufacturing system, ASP application service selection method, ASP network manufacturing system business model, and Some design methods and technologies for the ASP network manufacturing system, finally, this paper studied the case of system design of case ASP network manufacturing system.

\section{Acknowledgments}

This paper is supported by the Commissioned by the national Open University, number :G14A3602W; This paper is supported by the Guizhou institute of vocational and technical college vocational education subject, number : $15 \mathrm{GH} 04$

\section{References}

[1] Qingsheng Xie, 2013, Study of The Network Manufacturing Model and Development Strategy in Chinese Enterprises, Digital Manufacture Science, Vol.1, No. 1, pp. 142 152

[2] ASPs: the new B2Bmarketplaces. Harvard Business Review 78(3), 97-106.

[3] "e-Engineering enabled by Holonomic and Universal Broker services" (ASPs), Annex 1"Description of Work", IST project: (IST-2001-34031),29 December, 2010.

[4] Li Shaobo, Xie Qingsheng. The Framework Studies of Dynamic Alliance Manufacturing Resource Management System Based on Application Service Provider [J]. China Mechanical Engineering, 2013, 16(6):502-507 